\title{
Measurement of the Four-Point Susceptibility of an Out-of-Equilibrium Colloidal Solution of Nanoparticles Using Time-Resolved Light Scattering
}

\author{
Claudio Maggi, ${ }^{1, *}$ Roberto Di Leonardo, ${ }^{2}$ Giancarlo Ruocco, ${ }^{3}$ and Jeppe C. Dyre ${ }^{1}$ \\ ${ }^{1}$ DNRF Centre "Glass and Time," Department of Sciences, IMFUFA, Roskilde University, P. O. Box 260, DK-4000 Roskilde, Denmark \\ ${ }^{2}$ CNR-IPCF, UOS Roma clo Dip. di Fisica Università "Sapienza," I-00185 Roma, Italy \\ ${ }^{3}$ Dipartimento di Fisica, Università di Roma "Sapienza", I-00185, Roma, Italy
}

(Received 14 January 2012; published 28 August 2012)

\begin{abstract}
The spatial fluctuations of the dynamics of a colloidal system composed of nanoparticles are probed by a novel experimental setup, which combines homodyne and heterodyne dynamic light scattering focused onto a micron-sized volume via a microscope objective. The technique is used to measure the four-point susceptibility of an aging colloidal suspension, revealing a breakdown of the Gaussian approximation for the correlation function of the scattered electromagnetic field. The deviation from the Gaussian approximation increases with waiting time as the system evolves toward an arrested phase, signaling the gradual emergence of higher-order nontrivial dynamic correlations.
\end{abstract}

PACS numbers: 78.40.Dw, 78.35.+c, 78.55.Bq, 78.67.Bf

Introduction.-Characterizing the complex behavior of soft condensed-matter requires the development of refined conceptual and experimental tools. Many systems of wide theoretical and practical interest like supercooled liquids, colloids, polymers, etc.—exhibit complicated structural and dynamic features. In particular, the motion of the constituting elements of these systems (atoms, macromolecules, monomers, etc.,) might be characterized by large spatial heterogeneities displaying large fluctuations in space. This implies that in some regions of the system the dynamics proceeds very slowly, while elsewhere it can be notably faster [1-3]. The experimental investigation of these features turns out to be very challenging, and few studies have established evidence of dynamic fluctuations significantly larger than the particles' size [4-6]. On the other hand, computer simulations have investigated intensively the heterogeneous scenario described above. Multipoint correlation functions and, in particular, the four-point susceptibility have been used to characterize the spatially heterogeneous dynamics in computer simulations $[7,8]$.

In this Letter, we present an experiment capable of measuring directly the four-point susceptibility in offequilibrium nanoparticle colloidal suspensions. In this case, the approximation schemes $[7,8]$ for the four-point susceptibility recently proposed, based on equilibrium properties, are not applicable since the system is aging. We measure directly the four-point susceptibility without resolving the positions of the individual particles. The experiment employs two different dynamic light-scattering techniques by combining heterodyne and homodyne photon-correlation spectroscopy (PCS). The idea of the experiment is to use a very small scattering volume in order to enhance the sensitivity in measuring dynamic fluctuations: the smaller the studied volume, the larger are the non-Gaussian components of the fluctuations. The experiment is performed on an aging low-concentration colloidal solution of Laponite, which is a synthetic clay that has been studied intensively for its rich dynamic and structural behavior $[9,10]$ as well as numerous applications [11]. Due to its simple preparation procedure, this colloidal system is useful for experimentally elucidating the basic physics soft materials with complex viscoelasticity [12].

Several features of these colloidal suspensions make them particularly suitable for studying off-equilibrium dynamics and for the present experiment. These colloids age spontaneously toward an arrested state after the preparation at room temperature and pressure (without requiring any fast cooling procedure). The suspension is easily prepared by stirring vigorously purified water with Laponite in the form of a powder. The aging process of the colloidal solution proceeds very slowly, ranging from several hours to days, depending on the concentration of the colloid in the solvent. We choose to study a low-concentration solution (concentration weight $C_{w} \approx 1.1 \times 10^{-2}$ ), which has a very slow aging toward the arrested phase. Because of their extremely small dimensions, Laponite particles cannot be directly imaged by optical microscopy, each colloid having the form of a flat cylinder with a thickness of about $1 \mathrm{~nm}$ and a radius of about $15 \mathrm{~nm}$. In the experimental setup presented below, complete information on the particle positions is not needed to measure directly the four-point susceptibility.

Experiment.-In a dynamic light-scattering experiment, one studies the dynamics of the light scattered by a medium. This radiation is selected to be directed along a specific propagation vector with a well-defined polarization [13]. We combine heterodyne and homodyne PCS measurements to determine the four-point susceptibility. This is done by setting up the experiment as depicted in Fig. 1. When the shutter is open, a portion of the laser radiation interferes on an optical-fiber beam splitter (BS2) 


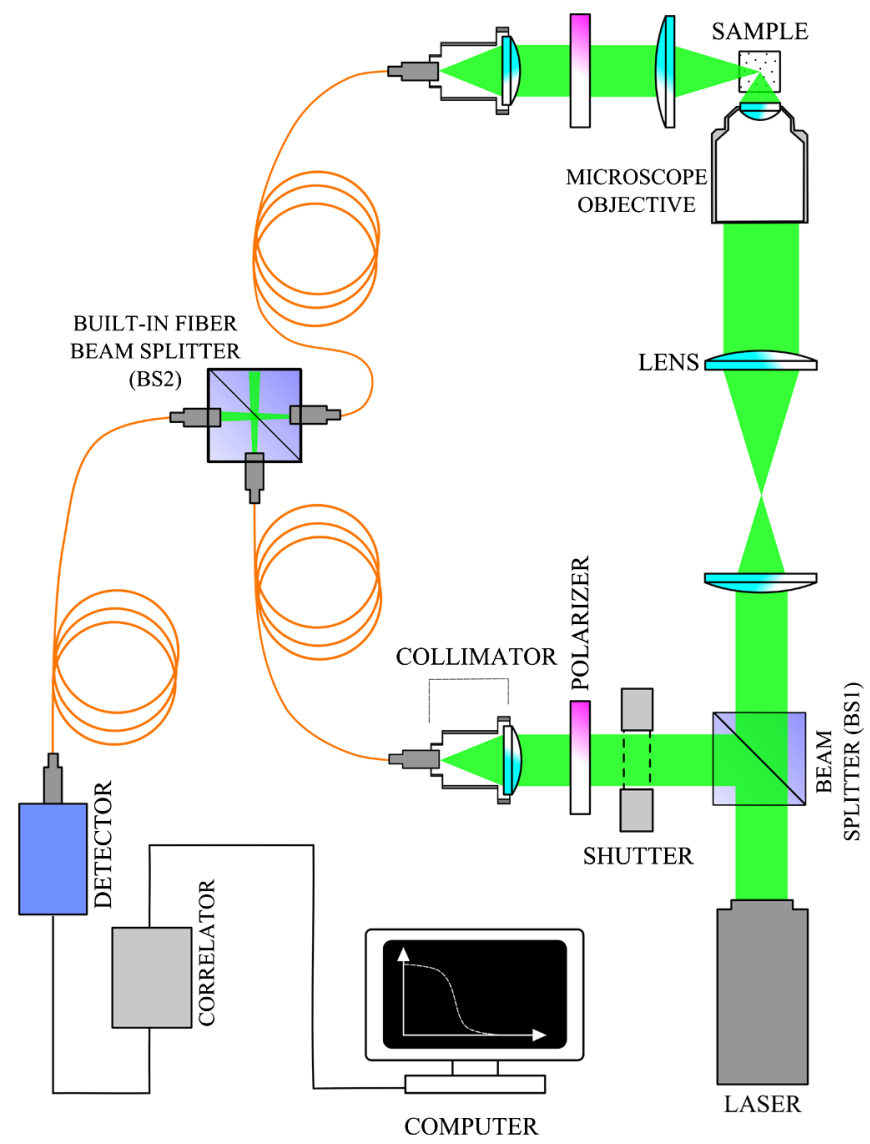

FIG. 1 (color online). Experimental setup for the combined measurement of the heterodyne and of homodyne correlation functions with a small scattering volume. The laser beam is focused onto the sample by a microscope objective. The scattered electromagnetic wave is collected by a lens and directed to the optical fiber system. If the shutter is open, the laser field is mixed with the scattered radiation by the built-in fiber (BS2) and delivered to the detector. In this case, the heterodyne function is measured. If the shutter is closed, a homodyne measurement is performed.

with the light scattered by the sample, setting the experiment in the heterodyne mode. Measuring the autocorrelation function of the intensity of these interfering fields, we obtain a correlation function that is proportional to the time-correlation function of the scattered electric field $E(t)$ :

$$
C_{1}\left(t_{w}, t\right) \propto\left\langle E^{*}\left(t_{w}\right) E(t)\right\rangle,
$$

where $t_{w}$ is the aging time. Notice that the instantaneous value of the field coincides with its fluctuation $E(t)-$ $\langle E(t)\rangle=\delta E(t)$ because $\langle E(t)\rangle=0$. The measured scattered field is directly related to the degrees of freedom of the colloidal platelets via $E(t) \propto \sum_{j}^{N} \alpha\left(\theta_{j}(t), \phi_{j}(t)\right) e^{i \mathbf{k} \cdot \mathbf{r}_{j}(t)}$, where $\alpha$ is the polarizability tensor component that depends on the orientation angles $\left(\theta_{j}, \phi_{j}\right)$ of the $j$ th Laponite disc, $\mathbf{r}_{j}$ is the position of the center of mass of the colloid, and $\mathbf{k}$ is the scattering vector [13], while the sum runs over the $N$ particles in the scattering volume. When the shutter is closed, one detects the intensity of the light scattered by the sample only. In this case, we operate in the homodyne mode where one measures the correlation function of the intensity of the field scattered by the sample. From this measurement, we obtain the autocorrelation function of the fluctuations of the intensity $I(t)=|E(t)|^{2}$ of the scattered electromagnetic field (up to a constant factor):

$$
C_{2}\left(t_{w}, t\right) \propto\left\langle\delta I\left(t_{w}\right) \delta I(t)\right\rangle=\left\langle\delta\left|E\left(t_{w}\right)\right|^{2} \delta|E(t)|^{2}\right\rangle .
$$

The functions $C_{1}$ and $C_{2}$ are measured at different times by opening and closing the shutter. Since aging of the suspension proceeds very slowly, the functions measured during a short aging time interval can be combined for calculating the four-point function, quantifying the heterogeneous dynamics. The fourth-order susceptibility can be defined as the difference between the correlation functions Eqs. (2) and (1) (squared) (see Ref. [8] and Supplemental Material [14]):

$\chi_{4}\left(t_{w}, t\right)=N \frac{\left\langle\delta\left|E\left(t_{w}\right)\right|^{2} \delta|E(t)|^{2}\right\rangle-\left\langle\delta E^{*}\left(t_{w}\right) \delta E(t)\right\rangle^{2}}{\left\langle\left|E\left(t_{w}\right)\right|^{2}\right\rangle^{2}}$,

where $N$ is the average number of colloidal particles in the scattering volume. By inserting the microscopic definition of the scattered field into Eq. (3), one can find that $\chi_{4}$ includes correlations involving up to four different particles. More generally, however, $\chi_{4}$ represents the "variance" of the correlator $\mathcal{C}\left(t^{\prime}, t\right)=E^{*}\left(t^{\prime}\right) E(t) /\left\langle\left|E\left(t^{\prime}\right)\right|^{2}\right\rangle$ telling us how much the correlation function fluctuates around its average value. This means that, if the scattering volume is not too large compared to the typical extension of the dynamic correlations, a finite number (say $\mathcal{M}$ ) of independent scattering regions contributes to the field-field correlation function. Accordingly, the variance of the normalized correlator $\mathcal{C}$ is of the order of $\mathcal{M}^{-1}$, i.e., $\left\langle(\Delta \mathcal{C})^{2}\right\rangle \sim 1 / \mathcal{M}$. Being $\chi_{4}\left(t^{\prime}, t\right)=N\left\langle(\Delta \mathcal{C})^{2}\right\rangle$ [Eq. (3)], the four-point susceptibility is interpreted as the "dynamic amount of particles" contained in one of those correlated regions. If the scattering volume were too large, it would be difficult to experimentally resolve any significant difference between the fieldfield and the intensity-intensity correlation functions. In practice, measuring the four-point susceptibility amounts to measuring the deviation from the Gaussian approximation, according to which $\chi_{4}=0$ (as it is usually assumed in standard dynamic light-scattering experiments [13]). Consequently, we employ a focusing microscope objective (numerical aperture 0.25) and a short-focal collecting lens (focal length $35 \mathrm{~mm}$ ), which results in a micron-sized scattering volume (Fig. 1; see Supplemental Material [14] for more details).

Finally, it should be noticed that in order to arrive at $\chi_{4}$ as expressed by Eq. (3) from the experimentally measured functions, a few additional assumptions must be made: (i) The fluctuations of the total number of particles contained in the scattering volume gives a negligible 


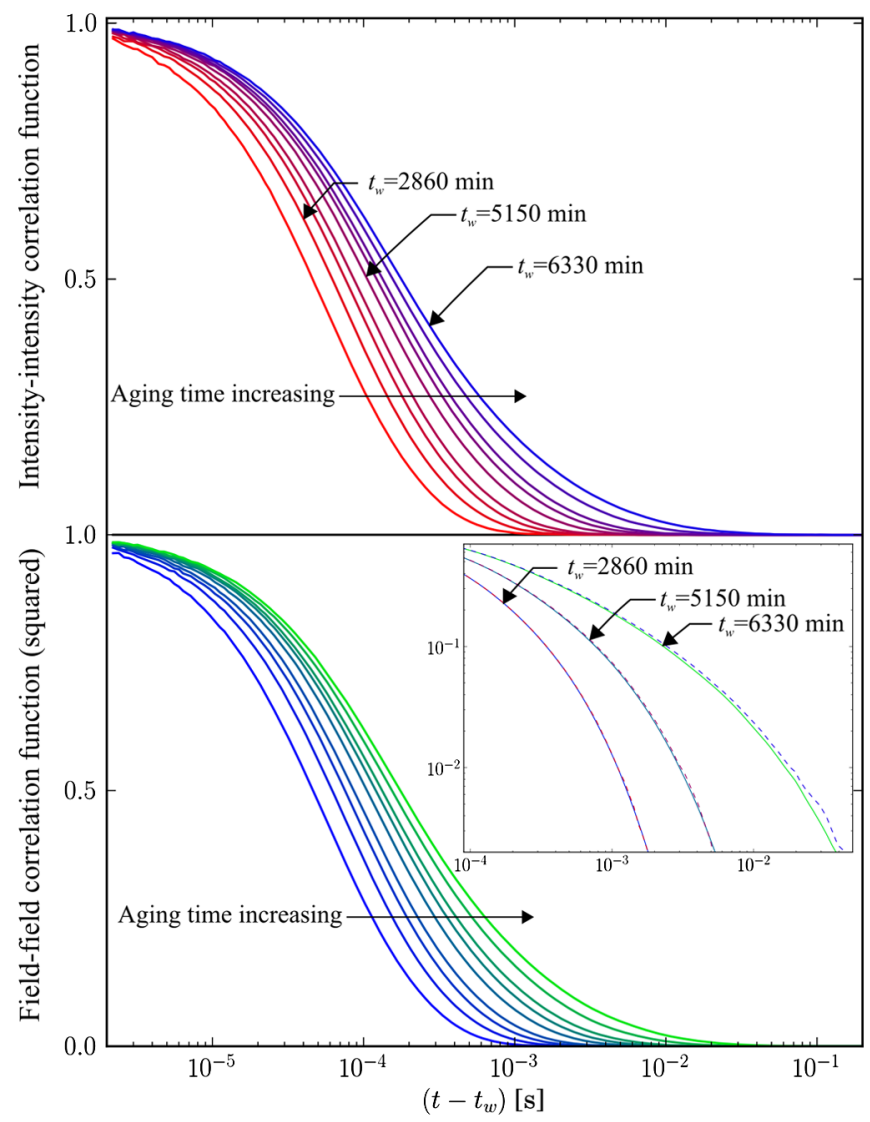

FIG. 2 (color online). Normalized field-field correlation function squared (top) and intensity-intensity correlation function (bottom) at several different aging times for a Laponite sample with $C_{w} \approx 1.1 \times 10^{-2}$. The field-field correlation function (full) and intensity-intensity (dashed) correlation function are well overlapped at short aging times, while at the longest aging time a small but significant separation between them is observed. This signals the failure of the Gaussian approximation and the emergence of higher-order correlations.

contribution to the dynamic susceptibility; (ii) any uniform flow along the scattering vector can be neglected in the measurement of the heterodyne correlation function; (iii) the deviation from the Gaussian approximation is small at $\left(t-t_{w}\right)=0$ so that it can be set to zero to a good approximation; this corresponds to forcing the homodyne correlation function to unity at $\left(t-t_{w}\right)=0$ as done in Fig. 2 (bottom) (see Supplemental Material [14] for a more detailed discussion of these three points).

Results.-As mentioned above, we obtain the intensityintensity correlation function from the homodyne measurement and the field-field correlation function from the heterodyne measurement. The two functions measured at several different aging times $\left(t_{w}\right)$ are reported in Fig. 2. A change in the correlation functions is evident, and as the system ages, these functions relax on longer and longer time scales. As seen in the inset of Fig. 2, these functions overlap very well at short aging times, while at large aging times the two are slightly separated, signaling the failure of the Gaussian approximation. Since the correlation

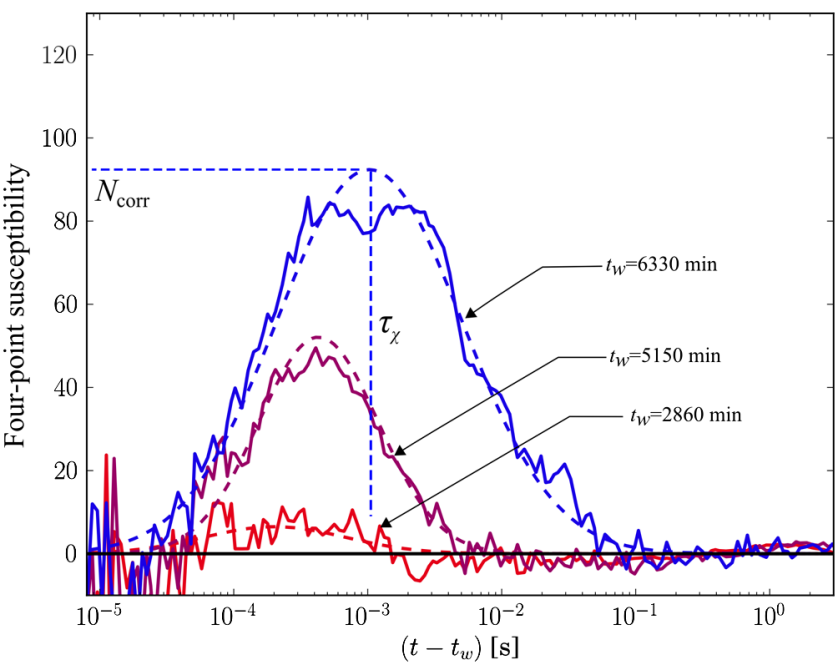

FIG. 3 (color online). Four-point susceptibility [Eq. (3)] at three well-separated aging times for a Laponite sample with $C_{w} \approx 1.1 \times 10^{-2}$. The function grows in amplitude, and its peak shifts to longer times as the aging time increases. A simple Gaussian function of $\ln \left(t-t_{w}\right)$ is used for fitting the data (dashed line). The maximum amplitude fitted allows a determination of the number of dynamically correlated colloidal particles $N_{\text {corr }}$.

functions are very stretched, it is convenient to define their relaxation time $\tau_{C}\left(t_{w}\right)$ as the integral of the correlation function $\tau_{C}\left(t_{w}\right)=\int_{0}^{\infty} d \Delta t\left\langle\delta I\left(t_{w}\right) \delta I(t)\right\rangle /\left\langle\left[\delta I\left(t_{w}\right)\right]^{2}\right\rangle$, where $\Delta t=\left(t-t_{w}\right)$. The growth of $\tau_{C}$ as a function of the aging time $t_{w}$ is shown in Fig. 4 (right). This is found to grow faster than exponentially with the aging time, confirming the findings of previous studies (see for example Ref. [9]).

The aging four-point susceptibility [Eq. (3)] is shown in Fig. 3. Despite the large noise in the signal, we find that $\chi_{4}$ clearly grows in amplitude; its peak shifts to longer times as the aging time increases. To characterize the amplitude and the position of the peak, we fit the data with a Gaussian function of $\ln \left(t-t_{w}\right)$. The maximum amplitude of the fourpoint susceptibility can be associated with the characteristic number of dynamically correlated colloidal particles in the relaxation of the scattered field $E(t)$, i.e., $\chi_{4}^{\max }\left(t_{w}\right)=$ $N_{\text {corr }}\left(t_{w}\right)$ (see Supplemental Material [14]). The growth of $N_{\text {corr }}$ with $t_{w}$ is shown in Fig. 4 (left). Interestingly, we find that $N_{\text {corr }}$ grows approximatively as the logarithm of the relaxation time $N_{\text {corr }} \sim \log \left(\tau_{C}\right)$, as shown in the inset of Fig. 4, which is similar to what was found from the indirectly estimated $\chi_{4}$ in molecular supercooled liquids [15]. The peak position of $\chi_{4}$, indicated by time $\tau_{\chi}$, grows as the aging time increases following the relaxation time $\tau_{C}$ associated with the two-point correlation function (Fig. 4, right).

Conclusions.-The experiment demonstrates that it is possible to observe a deviation from the Gaussian approximation and, thereby, for the first time establishes directly that the four-point susceptibility function grows during aging of a Laponite solution. The experiment was performed on an out-of-equilibrium suspension of colloidal nanoparticles for which the approximation schemes valid for equilibrium 

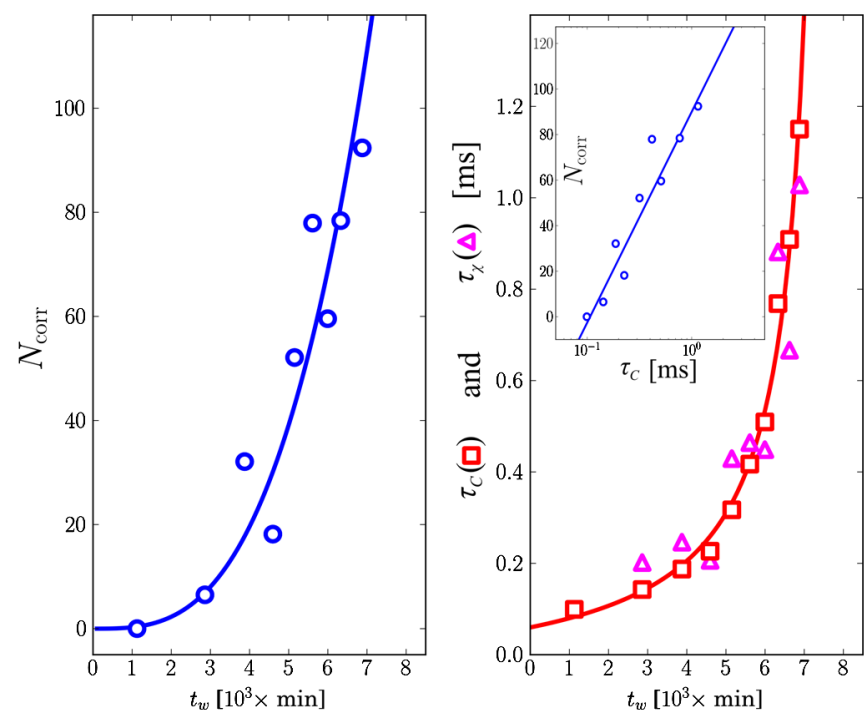

FIG. 4 (color online). (Left) $N_{\text {corr }}$ as a function of the aging time $t_{w}$ (the full line is a guide to the eye). (Right) Relaxation time $\tau_{C}$ associated with the two-point correlation function (squares; the full line is a guide for the eye). $\tau_{\chi}$ (triangles) is the peak position of the four-point correlation function as a function of $t_{w}$. It is seen that $\tau_{C}$ and $\tau_{\chi}$ grow in the same manner as $t_{w}$ increases. The inset shows $N_{\text {corr }}$ as a function of $\tau_{C}$ (logarithmic scale) where the straight line is a logarithmic fit of the data $\left(N_{\text {corr }} \sim \ln \tau_{C}\right)$.

supercooled liquids are not applicable. Direct imaging of the particles is not possible because of their extremely small dimensions. Nevertheless, we are able to access $\chi_{4}$ by measuring two correlation functions in a small scattering volume. It is important to note that our approach does not require one to confine the liquid in a small volume, which would induce surface effects that would inevitably affect the dynamic properties of the system studied in comparison to its bulk dynamics. In our experiment, by reducing the laser beam waist, we reduce the size of the probed volume. However, the size of the scattering volume, which can be reached with visible light, is restricted to the micron range. It seems quite possible that by using nano-focused $\mathrm{X}$ rays, a similar approach can be used for measuring directly the four-point susceptibility in molecular supercooled liquids and glasses. In the recent years, major advances have been made in $\mathrm{X}$-ray optics, making it possible to obtain beams with a waist down to $\sim 10 \mathrm{~nm}$ (see for example Ref. [16]). Moreover, X-ray PCS has been applied successfully to the study of a liquid metallic alloy (see Ref. [17]). For gel-forming systems, it would be also interesting to integrate our experiment with advanced methods [18] to measure structural properties and possibly find a link between static quantities and $\chi_{4}$. These seem to be promising routes to follow in the future study of the complex dynamics of supercooled liquids, gels, and glasses. *claudio.maggi@roma1.infn.it

[1] M. M. Hurley and P. Harrowell, Phys. Rev. E 52, 1694 (1995).

[2] C. Bennemann, C. Donati, J. Baschnagel, and S.C. Glotzer, Nature (London) 399, 246 (1999); C. Donati, S. Franz, S. C. Glotzer, and G. Parisi, J. Non-Cryst. Solids 307-310, 215 (2002).

[3] S. Whitelam, L. Berthier, and J. P. Garrahan, Phys. Rev. Lett. 92, 18 (2004)

[4] M. D. Ediger, Annu. Rev. Phys. Chem. 51, 99 (2000).

[5] U. Tracht, M. Wilhelm, A. Heuer, H. Feng, K. SchmidtRohr, and H. W. Spiess, Phys. Rev. Lett. 81, 2727 (1998); E. V. Russell and N.E. Israeloff, Nature (London) 408, 695 (2000); D. A. Vanden Bout and L. A. Deschenes, Science 292, 255 (2001).

[6] E. R. Weeks, J.C. Crocker, A. Levitt, A.C. Schofield, and D. A. Weitz, Science 287, 627 (2000); W. K. Kegel and A. van Blaaderen, Science 287, 290 (2000); P. Ballesta, A. Duri, and L. Cipelletti, Nature Phys. 4, 550 (2008).

[7] L. Berthier, G. Biroli, J. P. Bouchaud, L. Cipelletti, D. E. Masri, D. L'Hôte, F. Ladieu, and M. Pierno, Science 310, 1797 (2005).

[8] L. Berthier, G. Biroli, J.P. Bouchaud, W. Kob, K. Miyazaki, and D. R. Reichman, J. Chem. Phys. 126, 184503 (2007); L. Berthier, G. Biroli, J. P. Bouchaud, W. Kob, K. Miyazaki, and D. R. Reichman, J. Chem. Phys. 126, 184504 (2007).

[9] B. Ruzicka, L. Zulian, and G. Ruocco, Phys. Rev. Lett. 93, 258301 (2004); S. Jabbari-Farouji, G. H. Wegdam, and D. Bonn, Phys. Rev. Lett. 99, 065701 (2007).

[10] C. Maggi, R. Di Leonardo, J. C. Dyre, and G. Ruocco, Phys. Rev. B 81, 104201 (2010).

[11] P. Labbé, B. Brahimi, G. Reverdya, C. Moustyb, R. Blankespoor, P. Labbé, B. Brahimi, A. Gautier, and C. Degrand, J. Electroanal. Chem. 379, 103 (1994); C. Galez, J. Teyssier, R. Le Dantec, C. Galez, Y. Mugnier, J. Bouillot, and Jean-Claude Plenet, Appl. Phys. Lett. 85, 710 (2004); I. Boucenna, L. Royon, and P. E. Colinart, J. Therm. Anal. Calorim. 98, 119 (2009).

[12] F. Ianni, S. Gentilini, and G. Ruocco, Phys. Rev. E 75, 011408 (2007); F. Ianni, R. Di Leonardo, S. Gentilini, and G. Ruocco, Phys. Rev. E 77, 031406 (2008).

[13] B. J. Berne and R. Pecora, Dynamic Light Scattering (Dover Publications Inc., New York, 1975).

[14] See Supplemental Material at http://link.aps.org/ supplemental/10.1103/PhysRevLett.109.097401 for the estimate of the scattering volume and for a discussion of the assumptions made.

[15] C. Dalle-Ferrier, C. Thibierge, C. Alba-Simionesco, L. Berthier, G. Biroli, J.-P. Bouchaud, F. Ladieu, D. L'Hôte, and G. Tarjus, Phys. Rev. E 76, 041510 (2007).

[16] H. C. Kang, C. Liu, J. Maser, G. B. Stephenson, R. Conley, A. T. Macrander, and S. Vogt, Phys. Rev. Lett. 96, 127401 (2006).

[17] M. Leitner, B. Sepiol, L.-M. Stadler, B. Pfau, and G. Vogl, Nature Mater. 8, 717 (2009).

[18] B. J. Ackerson, T. W. Taylor, and N. A. Clark, Phys. Rev. A 31, 3183 (1985). 